\title{
Analysis of the dependence of stresses and deformations on the surfaces of shoe soles of different thicknesses and materials
}

\author{
Olesya Golubeva $^{1^{*}}$ and Alina Pogorelova ${ }^{1}$ \\ ${ }^{1}$ Don State Technical University, Gagarina sqr., 1, 344003 Rostov-on-Don, Russia
}

\begin{abstract}
For this study, three-dimensional models of the soles of work shoes of different thicknesses and consisting of different materials were created. These models were analyzed to obtain the distribution of stress and strain on their surfaces. To build the model, we used experimental data on the shape and size of the sole, as well as the properties of specific materials used in the manufacture of soles for work shoes used in agriculture. The goal was to determine the most suitable material, that is, which of the materials has the most suitable characteristics for the sole, has the best wear resistance when used in agriculture. We noticed a significant reduction in deformations on the surface of the sole with an increase in the thickness of the sole, as well as when using two-component casting of the sole from a combination of materials: low-density EVA and flexible PU, medium-density EVA and soft PVC. This indicates their advantages and maximum suitability.
\end{abstract}

\section{Introduction}

Modern work shoes are an important element of a worker's equipment. This is both the protection of the worker's legs, and a means of the normal functioning of the entire body during the working day, which should also have certain aesthetic properties.

Work shoes are a kind of personal protective equipment that reduces the risk of occupational injuries. In this regard, shoes should be protected from bumps, punctures, low and high temperatures, from slipping, water, petroleum products, acids, alkalis, etc. These are serious requirements for a product that must perform its functions during a certain period of its operation.

Reliability is one of the most important indicators of the quality of shoes. Reliability refers to the properties of the product to perform specified functions, maintaining its performance within certain limits under specified operating modes and conditions of use, as well as during storage.

Reliability includes indicators of reliability, durability, maintainability and persistence. Reliability indicators characterize the property of the product to continuously maintain its performance for some time or some operating time. For the shoes in question, these indicators will be of interest to us in the first place. To achieve these goals, it is advisable to consider shoes as a system.

\footnotetext{
*Corresponding author: 1354565@mail.ru
} 
Of great importance for the performance of the system is not only its internal structure (for shoes-design), including the characteristics of individual elements, but also external influences or conditions in which this system works.

Software modeling of shoes allows us to create not only an accurate prototype of the product, but also is a tool in the design and functional evaluation of the product $[1,3]$. One of the most popular approaches to shoe modeling is based on the finite element method (FEM). Most studies are aimed at studying and analyzing the bottom of the shoe, the top of the product is rarely considered. Finite element analysis contributes to the experimental approach to obtain preliminary data on the distribution of loads on the foot and on the product itself [2-6].

As a rule, the design of the sole is simple and is not based on scientific data, but on fashion. But in addition to the material and shape, the distribution of stress on the surface of the sole is significantly affected by its thickness. The thicker it is, the more effective it is [9$12]$.

Finite element analysis allows you to effectively evaluate the design and material characteristics of shoes even before they are made. The analysis can be carried out to compare the effects on the surface of the sole, when changing its thickness, stiffness or material [3].

\section{Method}

The purpose of this study is to create a three-dimensional model of the sole, on the basis of which an analysis will be carried out to obtain a picture of the distribution of stresses and deformations on its surface.

The theoretical and methodological basis of the research was the theory of complex systems modeling, the theory of operations research, and the method of system-structural analysis of objects.

To implement this, data on the shape of the sole and the properties of the materials under study were obtained. The goal is to identify the most suitable materials for the sole. The construction of the sole model, as well as the analysis, was carried out using the Ansys software.

It is assumed that the materials are elastic-plastic, isotropic, and homogeneous.

According to the literature and numerous tests of recent years, ethylvinyl acetate (EVA) can be used for the manufacture of soles. The shock-absorbing properties of the product will depend on its density. EVA deformations are linear and studies show that the density decreases with increasing strain strength. It was also found that the pressure in the heel area is reduced by adding components based on medium-density polyurethane(PU), mediumdensity EVA and low-density EVA. A similar situation is observed in the area of the metatarsal bones. Many studies provide data on the effectiveness of the use of soft polyvinyl chloride(PVC) in this matter $[1,7,8]$.

The novelty of this study is the combination of materials to produce soles of different thicknesses.

Based on the literature data, the following materials and their properties are considered:

Table 1. Materials and their properties.

\begin{tabular}{|c|c|c|c|c|}
\hline & $\begin{array}{c}\text { EVA low } \\
\text { density }\end{array}$ & $\begin{array}{c}\text { EVA } \\
\text { medium } \\
\text { density }\end{array}$ & $\begin{array}{c}\text { PVC } \\
\text { soft }\end{array}$ & PU flexible \\
\hline Density $\left(\mathrm{g} / \mathrm{cm}^{3}\right)$ & 0,923 & 0,941 & 1,245 & 1,250 \\
\hline $\begin{array}{c}\text { Young Modulus } \\
(\mathrm{MPa})\end{array}$ & 11 & 60 & $20-50$ & 60 \\
\hline
\end{tabular}




\begin{tabular}{|c|c|c|c|c|}
\hline $\begin{array}{c}\text { Poisson } \\
\text { Coefficient }\end{array}$ & 0,49 & 0,49 & 0,40 & 0,40 \\
\hline $\begin{array}{c}\text { Tensile Yield } \\
\text { Strenght (MPa) }\end{array}$ & 2,5 & 7,31 & 20 & - \\
\hline $\begin{array}{c}\text { Tensile Ultimate } \\
\text { Strenght (MPa) }\end{array}$ & 1,9 & 12,5 & 15,8 & $20-30$ \\
\hline
\end{tabular}

Since our analysis is static, the body weight is distributed evenly on both legs. The load force is: $\mathrm{G}=\mathrm{m}^{*} \mathrm{~g}$, where $\mathrm{G}$ is the force of gravity, $\mathrm{m}$ is the body weight (in this study, the body weight is $60 \mathrm{~kg}), \mathrm{g}$ is the acceleration of gravity $(9.81 \mathrm{~m} / \mathrm{s} 2)$.

\section{Results}

Using the Ansys software, a model of a sole with a thickness of $10 \mathrm{~mm}$ and a heel of $20 \mathrm{~mm}$ was created.

The results of the calculation of equivalent stresses (von Mises stresses) showed an increase or decrease in deformations depending on the material of the sole. Detailed data on the change in the equivalent stress with the change in the thickness of the sole of different materials are shown in Figures 1-6.

According to the results of using a two-layer EVA low density + EVA medium density sole (fig.1), an increase in the thickness of the sole leads to a significant increase in the Equivalent Von-Misses stress.

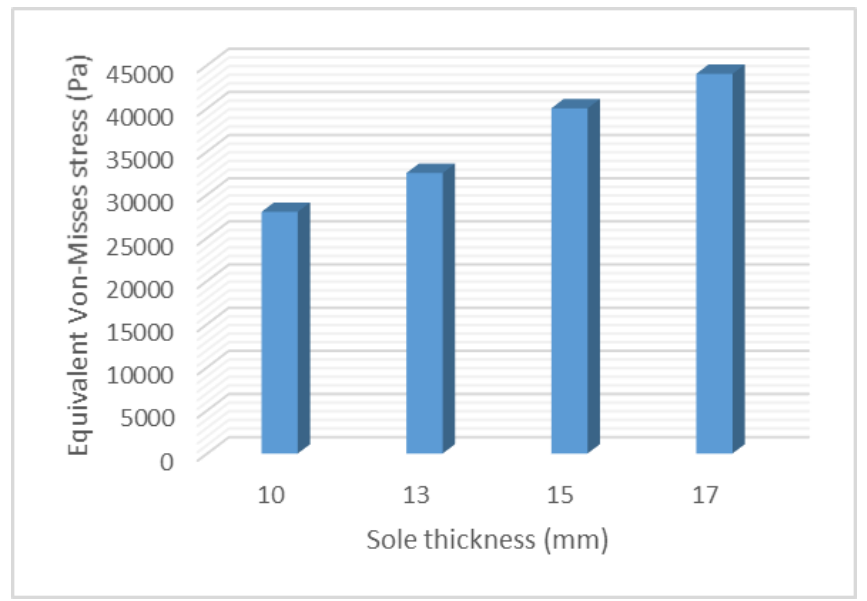

Fig. 1. EVA low density + EVA medium density

According to the results of using a two-layer EVA low density + PU flexible sole (fig.2), an increase in the thickness of the sole leads to a significant reduction in the Equivalent VonMisses stress. 


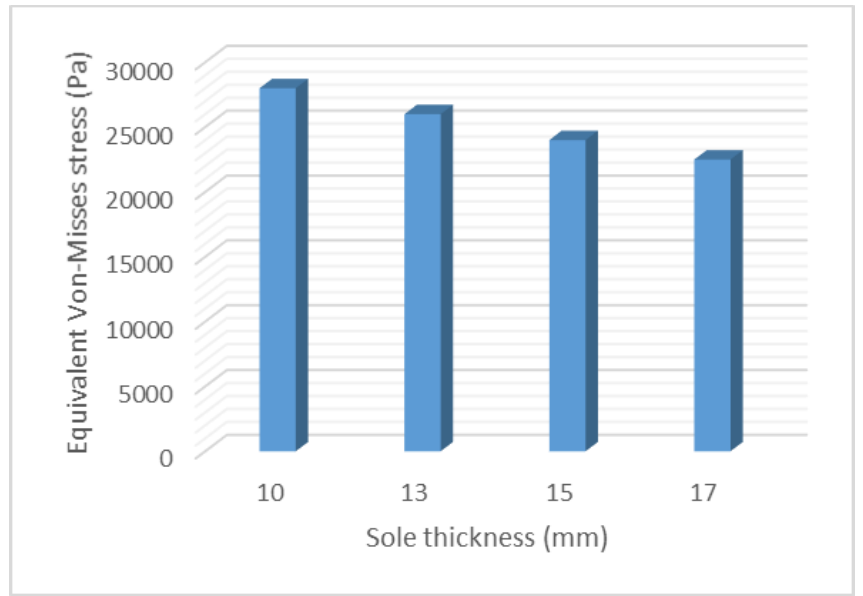

Fig. 2. EVA low density + PU flexible

According to the results of using a two-layer EVA low density + PVC soft sole (fig.3), an increase in the thickness of the sole leads to a significant increase in the Equivalent VonMisses stress.

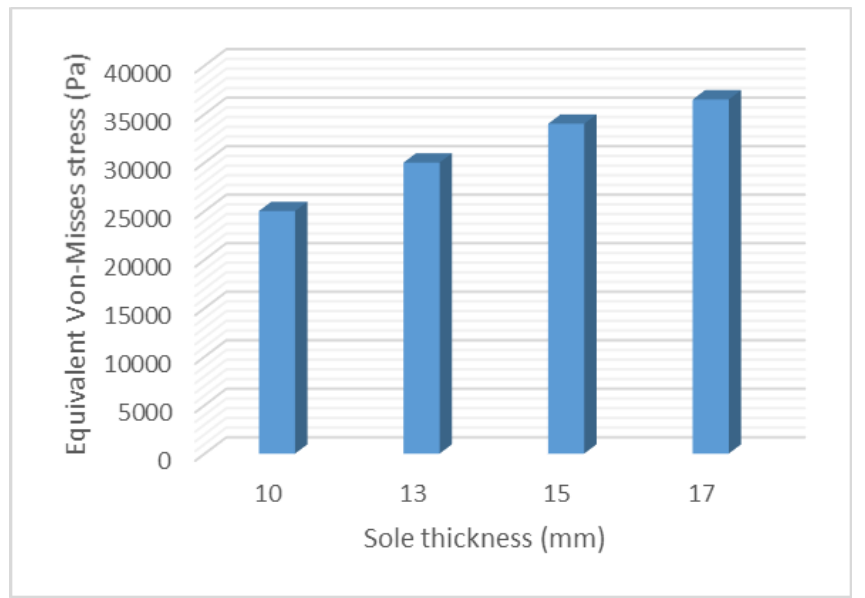

Fig. 3. EVA low density + PVC soft

According to the results of using a two-layer EVA medium density + PU flexible sole (fig.4), an increase in the thickness of the sole leads to a significant reduction in the Equivalent VonMisses stress. 


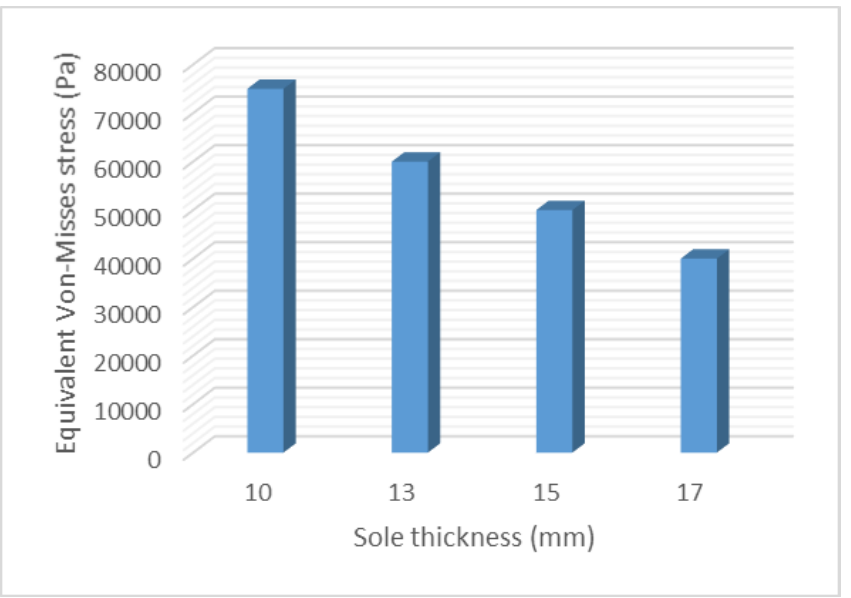

Fig. 4. EVA medium density + PU flexible

According to the results of using a double-layer EVA medium density + PVC soft sole (fig.5), an increase in the thickness of the sole leads to the most significant reduction in the Equivalent Von-Misses stress.

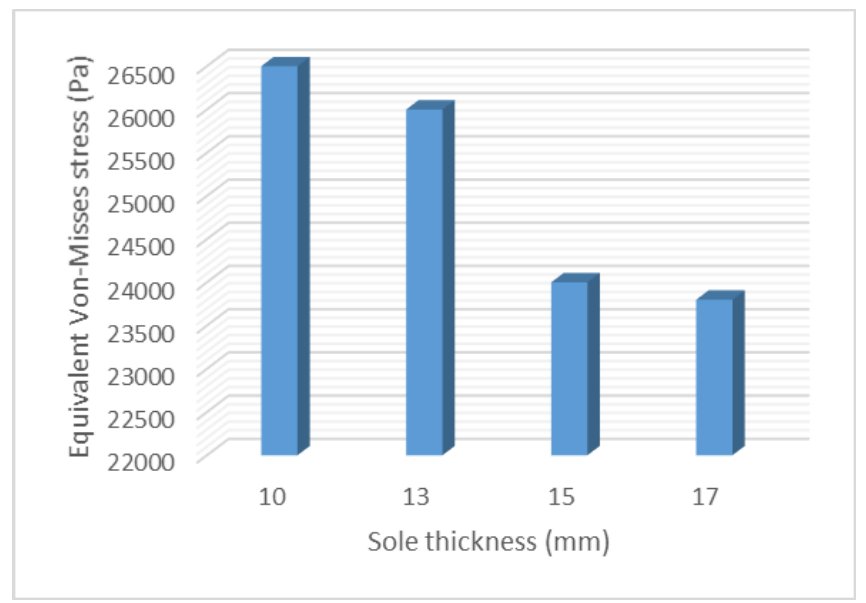

Fig. 5. EVA medium density + PVC soft

According to the results of using a double-layer PVC soft + PU flexible sole (fig.6), an increase in the thickness of the sole leads to a less significant reduction in the Equivalent Von-Misses stress. 


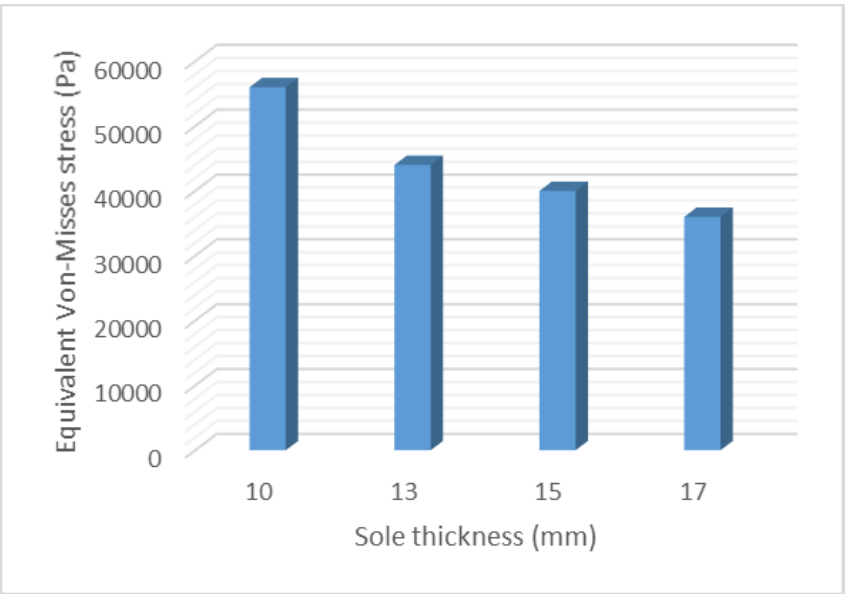

Fig. 6. PVC soft + PU flexible

The obtained data are confirmed by experimental data on the parts of the product that are subject to an increased degree of wear of the material during the working process [13-15].

\section{Conclusions}

A significant reduction in the stress on the sole with an increase in the thickness of the sole was found with a combination of materials: low-density EVA+PU and flexible mediumdensity EVA+PVC soft.

Given these results, when making the bottom of work shoes, a combination of materials that reduce stress on the surface is recommended [16].

In the case of a combination of low density EVA+EVA medium density is observed to increase the voltage while increasing the thickness of the soles, and in the case of soft PVC+flexible PU reduction efforts with increasing thickness is observed, but the values are still higher than the recommended combinations.

Based on the finite element method, it can be concluded that changes in the thickness, density, or material of the sole can significantly redistribute the stresses and deformations on the surface of the sole during the operation of work shoes in agriculture.

It is necessary to focus on one more problem - the equal strength of the elements of the shoe design. For work shoes, this is important, since the time of its operation is regulated by regulatory documents. Modern materials and technologies allow you to do this. The whole problem lies in the fact that it is not yet well enough and adequately possible to evaluate and predict the performance properties of materials by laboratory methods at the stage of preparation for the production of shoes due to the lack of measurement tools and a regulatory framework.

\section{References}

1. K. Brueckner, J. Heidnefelder, S. Odenwald, T.L. Milani "Mechanical and biomechanical characterization of running shoes with different midsole materials before and after aging", Footwear Science 3(S1), 18-19 (2011)

2. V.N. Kraev, Management Decision Making Methods (2014)

3. J.C. Choi, C. Kim, J. Mater. Process Technol., 110 (2013) 
4. G. Colombo, D. Ferretti, U. Cugini, Proceedings of international symposium on advanced geometric modelling for engineering applications, 2-15 (2014)

5. G. La Rocca, L. Krakers, M.J.L. van Tooren, Proceedings 9th symposium on multidisciplinary analysis and optimization, 2-13 (2014)

6. A. Luximon, Handbook of Footwear Design and Manufacture 64(5), 416 (2013)

7. A. Healy, D. Dunning, N. Chockalingam, "Effect of insole material on plantar pressure”, Footwear Science 3(S1), 69-70 (2011).

8. T. Nishiwaki, J. Tateishi, "Basic research on midsole material designing", Footwear Science 3(S1), 122-123 (2011)

9. K. Brueckner, J. Heidnefelder, S. Odenwald, T.L. Milani, "Mechanical and biomechanical characterization of running shoes with different midsole materials before and after aging", Footwear Science 3(S1), 18-19 (2011)

10. Y.M. Tang, K.-C. Hui, "Human foot modeling towards footwear design", ComputerAided Design 43, 1841-1848 (2011)

11. A. Healy, D. Dunning, N. Chockalingam, "Effect of insole material on plantar pressure", Footwear Science 3(S1), 69-70 (2011)

12. T. Nishiwaki, J. Tateishi, "Basic research on midsole material designing", Footwear Science 3(S1), 122-123 (2011)

13. L. A. Ginis, Statistical Methods of Quality Control and Management, Applied Software (2019)

14. A.K. Ershov, Quality Management (2017)

15. S.I. Solonin, Control Charts Methods (2014)

16. I.V. Maruseva, Modern Management, Classic and Applied Aspects (2018) 